\title{
INTRODUCTORY LECTURE
}

\author{
Martin J. Rees \\ Institute of Astronomy \\ Madingley Road \\ Cambridge CB3 OHA \\ ENGLAND
}

It is now 22 years since quasars were discovered. When the ageing veterans of those pioneering investigations think back over two decades of boisterous debate, their reactions are probably rather mixed. Wonderment at the range and variety of novel phenomena revealed in all wavebands must be tinged with disappointment that we seem so slow in grasping what is really going on. Our understanding has advanced slowly, through many small steps - forward steps preponderating (fortunately) over backward ones.

Only gradually did quasars become generally recognised as just extreme instances of the bewildering variety of phenomena associated with active galactic nuclei. This zoo of objects spawned a confusing nomenclature: quasars, BL Lacs, blazars, optically violent variables (OVVs), Seyferts 1 - 2, starbursts, liners, and warmers. It might be logical to start off with definitions and taxonomy, but I doubt that this would really help. Let us just define a quasar as what Dr Swarup and his co- organisers deem to be one for the purposes of this conference, but recall S. Toulmin's dictum that "definitions are like belts: the shorter they are the more elastic they must be".

It is still premature to propose a multiparameter classification scheme with a real physical basis. Meanwhile, we may as well stick with the established nomenclature, "historical baggage" though it may be. All that matters is that we should agree on what the words mean, and be mindful that many characteristics (for instance, whether or not images appear "stellar") depend on the sensitivity of our instruments, being neither intrinsic nor fundamental.

Figure 1 depicts the main phenomena manifested by quasars, with rough estimates (which in some respects are uncertain and modeldependent) of the associated length scales. I went through the provisional programme of this meeting trying to classify the papers in terms of the relevant lengthscale, assigning this according to the theoretical prejudices embodied in Figure 1 . There are 23 papers dealing with scales $\sim 10^{15} \mathrm{cms}$ ("prime movers", X-ray and UV continuum), 48 papers with scales $\sim 10^{19} \mathrm{cms}$ ((broad emission lines and VLBI radio), 15 papers with $\sim 10^{23} \mathrm{cms}$ (fuzz and extended radio 


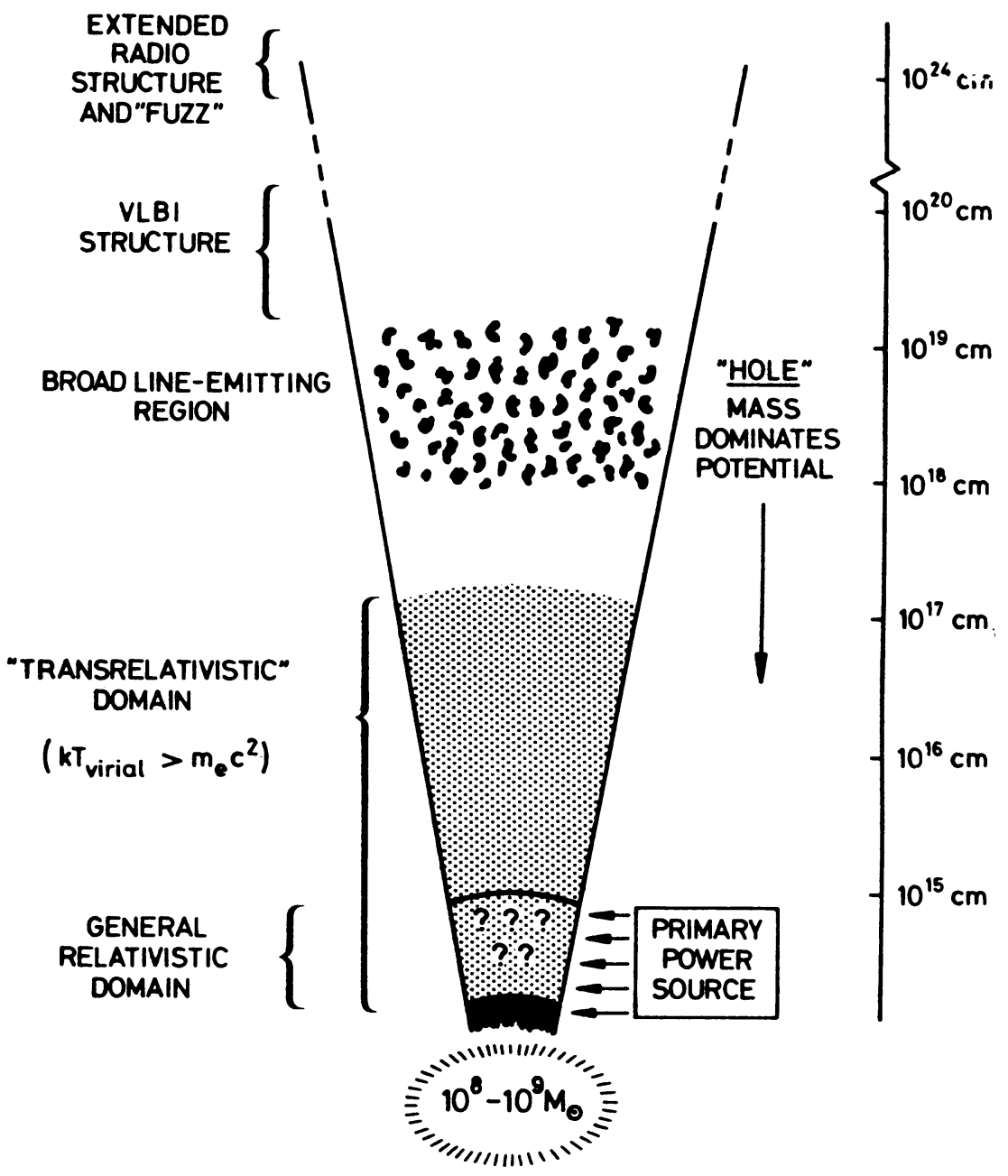

Figure 1. A schematic "slice" through a quasar, illustrating that observed phenomena span a range of almost $10^{10}-1$ in lengthscale. If the primary power source involves a single mass of $10^{8}-10^{9} \mathrm{M}_{\Theta}$, this object dominates the gravitational field (yielding a potential well of "1/r" form) out to a few parsecs. On larger scales, interaction of radiation and outflowing matter with the environment of the host galaxy is the determinant factor. General relativistic effects would be important within a few times $r_{s}$ (where $r_{s}=3 \times 10^{13}\left(M / 10^{8} M_{0}\right) \mathrm{cm}$ ); the primary power supply may be concentrated within this domain. Within a radius $\left(m_{p} / m_{e}\right) r_{s} \cong 10^{3} r_{s}$, the virial temperature would exceed the rest mass energy of an electron. 
structure); finally 30 papers address cosmological effects clustering, absorption lines, lensing, and cosmological evolution (i.e. scales $\sim 10^{27} \mathrm{~cm}$ ). Let us hope that this indeed proves to be the most useless statistical data presented at this conference! It is my prerogative as opening speaker to raise questions rather than necessarily answer them. I shall do this by highlighting, in a qualitative and subjective way, some issues which I hope we shall focus on during the week. Let me apologise in advance for omitting references or attribution for the various ideas mentioned here. Useful compilations, and benchmarks against which we can assess current progress, are the proceedings of five recent conferences concerned with quasars: those held at Liège (Swings 1983), Munich (Brinkman and Trumper 1984), Manchester (Dyson 1985), Santa Cruz (Miller 1985) and Trieste (Giuricin et al. 1986).

\section{THE EMISSION LINES ETC.}

It is unsurprising to find so many papers dealing with the broad emission lines - undoubtedly the most intensively studied aspect of quasars. There is a substantial body of data with high signal-tonoise, and modelling rests on the firm basis of conventional and well-understood physics. Among the still-unsettled questions are the following.

(a) Is photoionization by a UV continuum the only heat input?

(b) How well-defined is the radius of the broad line region and the cloud density within it? The standard density normally quoted is of order $10^{10}$ or $10^{11 \mathrm{~cm}^{-3}}$. But how much gas could be present with densities below $10^{9} \mathrm{~cm}^{-3}$ or above $10^{11} \mathrm{~cm}^{-3}$ ? Maybe those theorists who aim to show why clouds only condense at one characteristic radius are trying harder than they need.

(c) What is the spatial configuration? Is the gas in an approximately spherical distribution; or is it, on the other hand, in some kind of thick disc, or in a jet-like outflow?

(d) What are the kinematics? Is the gas flowing out in a wind, and if so what pushes it? Are clouds on gravitationally bound orbits? Or are they falling inward, perhaps as part of some accretion flow pattern?

Further issues, involving somewhat less direct inferences from observations, concern the formation of the inferred clouds and filaments, and the relation of the broadline region to the VLBI radio structure, which has comparable size.

Before "homing in" on the central engine $\left(\approx 10^{15} \mathrm{~cm}\right)$ note that many complexities may occur on intermediate scales $10^{15}-10^{18} \mathrm{~cm}$. A massive central object would dominate the gravitational field throughout this volume. The spatial distribution of the material would be as intricate as on any larger scale (though of course we have little hope of resolving it directly). One would expect, moreover, a multiphase structure: optically thick clouds at $10^{4}-10^{5} \mathrm{o} \mathrm{K}$, embedded in plasma where all the electrons may be marginally relativistic, and the ion kinetic temperature $\geq 1 \mathrm{Mev}$. The density of $>1 \mathrm{Mev}$ photons may be 
high enough for $\gamma+\gamma \rightarrow e^{+}+e^{-}$collisions to maintain a pair density at least comparable with the original electron density. Processes on these length-scales may be crucial in determining the emergent continuum spectrum (including a UV bump?) and in establishing the collimation of jets. The continuum emission mechanism may be quite different in a typical radio quiet quasar from in an OVV - only in the latter objects do we really know (from polarization, variability etc) that synchrotron-type radiation dominates.

The degree of directionality of the power output is another key question. There is good evidence for relativistic beaming on scales 1 - 10 parsecs from VLBI measurements of superluminal sources. More controversial, however, is the relevance of relativistic beaming to the demarcation between radio loud and radio quiet quasars, and the issue of whether BL Lacs and OVVs are beamed towards us. Quantitative details of the beaming would be sensitive to the flow pattern, and to shocks, etc., in the outflow. Beaming is directly observed on scales of parsecs and larger; it is still unclear whether the collimation is established only on that scale, or on the dimensions of the central source (see Figure 1).

Some directionality could arise merely from non-spherical geometry (even if there are no relativistic motions or large doppler effects). For instance, optically thick discs would appear brighter viewed face-on, and edge-on objects may appear faint because of absorption in a larger-scale disc. These issues could be clarified by seeking correlations between emission line widths and other properties.

\section{THE CENTRAL POWER SUPPLY}

At the first Texas conference, held in 1963, the implications of quasars for theorists were already recognised. As T. Gold said in his banquet speech on that occasion "Relativists and their sophisticated work are not only magnificent cultural ornaments, but might actually be useful to science!" (see Robinson et al. 1964). And one of the few opinions about quasars where the consensus has been steady since 1963 is that the energy is gravitational in origin. (An un- weighted majority vote is in itself, however, worth little in this subject!) Conditions in the central continuum-emitting region are more extreme, in terms of energy densities, etc., than in the emission line clouds (though the physics is by no means as exotic as on the surfaces of neutron stars, for instance).

The prime reason for invoking collapsed objects is that any gravitationally-powered source which releases more than $1 \%$ of its rest mass energy contracts unstoppably; and a collapsed object, once formed, offers a more powerful and efficient power source than any precursor system. So if the power is gravitational in origin, it is inconsistent not to envisage that collaped objects power the most powerful active nuclei - quasars and radio galaxies in particular.

Irrespective of the details of the central mechanism, there are simple thermodynamic constraints on the form of the primary emergent 


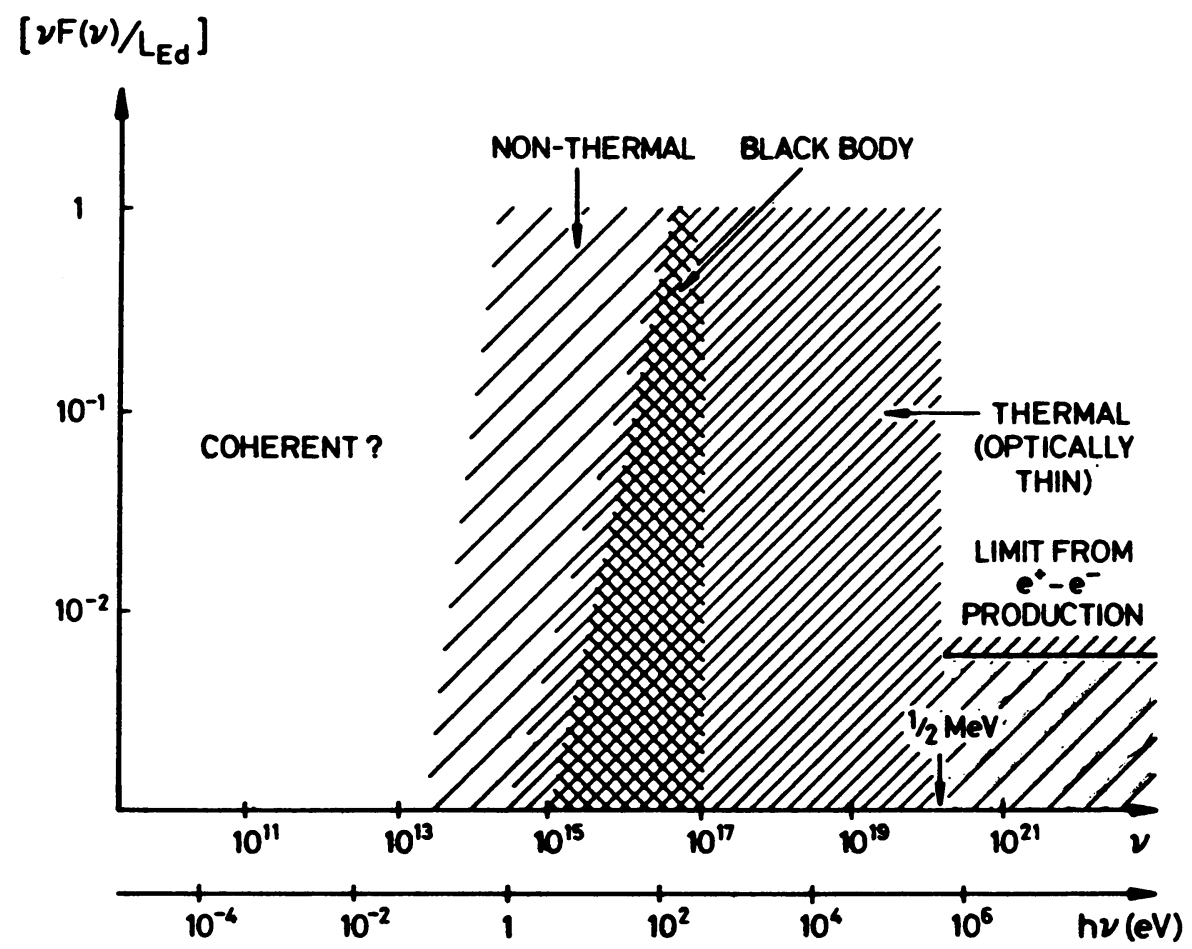

Figure 2. This diagram illustrates schematically the thermodynamic constraints on the radiation spectrum that could emerge directly from a source of luminosity $\mathrm{L}_{\mathrm{Ed}} \cong 10^{46} \mathrm{erg} \mathrm{s}^{-1}$ and dimensions $\sim 10^{14} \mathrm{~cm}$. Black body radiation with $\mathrm{T}_{\mathrm{bb}} \cong 3 \times 10^{5} \mathrm{~K}$ (heaviest shading) would peak in the UV. Optically thin thermal radiation from plasma with $T>T_{b b}$ could emerge in the $X$-ray band, but absorption would still prevent a high fraction of the radiation from emerging in the optical band even though the Rayleigh-Jeans Law would be higher than for the black body by a factor $T / T_{b b}$ (intermediate shading). Synchrotron self-absorption yields a cut-off in the infra-red, so incoherent non-thermal radiation is restricted to the part of the diagram that is lightly shaded. (This is calculated assuming a magnetic field strength $\sim 10^{4} \mathrm{G}$, the equipartition value expected in radial accretion flow). Absorption of $\gamma$-rays via $\gamma+\gamma \rightarrow e^{+}+e^{-}$limits the luminosity above 1 Mev to $\leqslant 0.01 \mathrm{~L}_{\mathrm{Ed}}$. The main direct output from the central source would therefore be: (i) Thermal radiation in the UV or X-ray bands, (ii) non- thermal radiation, anywhere from the near infra-red to hard $X$-rays (but few $\gamma$-rays) and (iii) a relativistic $e^{+}-e^{-}$pair outflow. 
radiation. Figure 2 illustrates these for a typical quasar with $M=$ $10^{8} M_{\odot}, L_{E d} \cong 10^{46}$ erg $s^{-1}$ and dimensions $\sim 10^{14} \mathrm{~cm}\left(\sim^{2} r_{s}\right)$. If a luminosity $L_{E d}$ emerged with a black body spectrum, the temperature would be $\sim 3 \times 10^{5} \mathrm{~K}$, the flux therefore peaking in the far ultraviolet; a luminosity $\sim L_{E d}$ in the UV or $X$-ray band could be generated via an optically-thin thermal mechanism (e.g. Comptonised bremsstrahlung); even if the radiation were non-thermal, involving relativistic electrons, synchrotron self-absorption produces a cut-off in the infrared (implying, of course, that no radio emission can come from dimensions $\sim 10^{14} \mathrm{~cm}$ unless a coherent mechanism is operative); the pair production constraint would prevent more than $0.01 \mathrm{~L}_{\mathrm{Ed}}$ from emerging as $\gamma$-rays with energies above $1 \mathrm{Mev}$. These simple considerations would tell us immediately - even if we did not have other evidence - that the radiation we actually observe must be generated or reprocessed further out (see Figure 1).

Although there are genuinely compelling reasons for invoking generic "gravitational pits" in galactic nuclei, there is little evidence that the central objects have the distinctiveproperties of black holes in Einstein's theory - that they are, in other words, described by the Kerr metric. Theoretical models that postulate Kerr black holes are nonetheless interesting for two reasons. First, they may lead to a more quantitative and specific model for the form of the continuum radiation: the emergent energy could derive from accretion, or alternatively from electromagnetic effects that tap the holes' spin energy, converting it into non-thermal power and electromagnetic beams. Secondly, quasars may offer relativists a diagnostic that permits, for the first time, tests of general relativity beyond the weak-field approximation. Such modelling also involves other poorly-understood complications - for instance, relativistic MHD and radiation processes in pair-dominated plasmas.

\section{WHAT CAN WE REALISTICALLY EXPECT FROM THEORIES AND MODELS?}

How will we ever really know whether there are black holes in AGNs? The evidence can never be more than circumstantial. But we should not be too downcast by that. After all, the evidence that the Sun is powered by nuclear fusion, a cherished dogma never seriously contested, is also really just circumstantial. However, the confrontation of theory with observations, indirect even for stars, is more ambiguous still for quasars: in stars, energy percolates to the observable surface in a relatively steady, symmetric, and well-understood fashion; but in galactic nuclei it is reprocessed into all parts of the electromagnetic spectrum on scales spanning many powers of 10 , in a way that depends on poorly-known environmental and geometrical effects in the host galaxy.

The generic black hole model is not infinitely flexible, and not invulnerable. It could be refuted in at least three ways:

1 . by finding very regular periodicities, particularly on timescales below 1 hour. 
2. by showing that the central masses were $\ll 10^{6} \mathrm{M}_{\odot}$ in Seyferts or « $10^{8} \mathrm{M}_{\odot}$ in radio galaxies;

or 3 . by developing a theory of gravity more convincing than general relativity which prohibits black holes.

But a clean-cut refutation, leading to abandonment of some

theory, happens only rarely in astrophysics; many models have persisted unrefuted for a long time. A cynic might argue that they have survived only because they don't go beyond generalities, or else because their proponents have been adept, like the motor mechanics who must abound here in India, at replacing or modifying faulty parts to keep shaky old models "roadworthy". Such a cynical attitude is not necessarily justified, and to explain why I must digress briefly into methodology. The way we are told science is done is like this: the data suggest a model, which suggests further tests, whereby the original model is either refuted or refined. Such a simple scheme is realistic in, for instance, particle physics, where the fundamental entities may be exactly reducable to a few basic constants and equations. But other sciences deal with inherently complex phenomena and no theoretical scheme can be expected to account for every detail. In geophysics, for instance, the concepts of continental drift and plate tectonics have undoubtedly led to key advances; but they cannot be expected to explain the shape - the precise topography - of the continents. What we should aim to do, in our attempts to understand quasars, is focus on those features of the data which genuinely test crucial ideas, and not to be diverted into measuring or modelling something which is accidental or secondary.

The problem of interpretation can be illustrated by an (almost arbitrarily chosen) example: the data on continuum $X$-ray emission from quasars. As summarised in Figure 3, one can envisage various

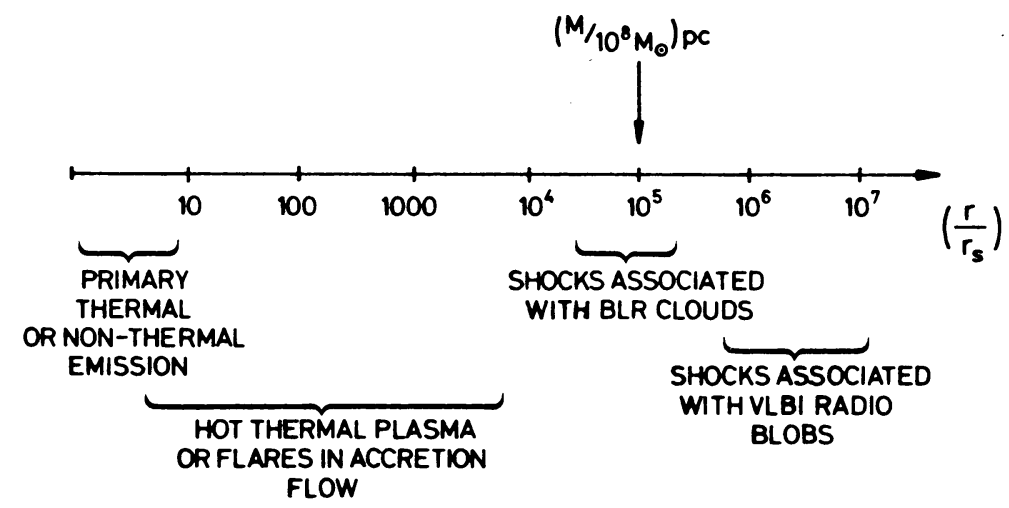

Figure 3. This diagram indicates that several different mechanisms, operating on quite different dimensions, could contribute to the observed $X$-ray continuum from quasars. The relative contribution from each mechanism could plausibly depend on radio properties, orientation, etc. (Absorption of soft $X$-rays by gas on $\mathrm{kpc}$ scales in the host galaxy could further complicate attempts to fit the data in terms of a few-parameter model.) 
processes, on very different scales, any of which could contribute significantly to the observed $X$-rays. This means that the overall $X$-ray spectrum is unlikely to qive a qood fit to any simple model and is in itself of limited interest. On the other hand, the detection of large amplitude rapid variability in the $X$-rays would clearly pin down the scale of the dominant emission, and thereby discriminate among the candidate production mechanisms mentioned in Figure 3 . Detection of line features would also, obviously, be an important discriminant.

When the phenomena being studied are inherently complicated and ambiguous, the confrontation between observation (or experiment) and theory is indirect. It is mediated by what one might call a scenario", like this:

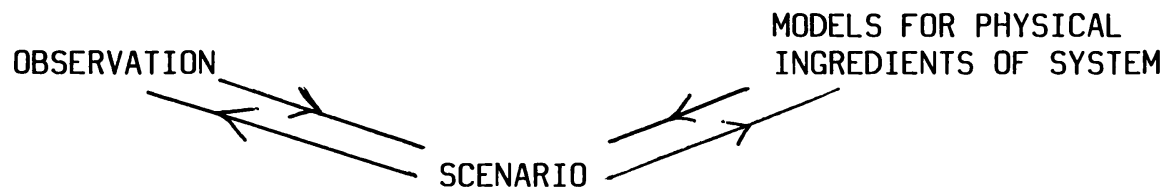

In the case of quasars, elements of the scenario include the things depicted in Figure 1 - clouds, inflow, winds, jets, radio blobs, shocks, collapsed objects, and so forth. We need some such scenario in our minds in order to interpret observations, and formulate ideas for further observations. A quasar is too complicated, and involves far too many parameters, to be quantitatively modelled all in one go; the scenario nevertheless suggests what the essential ingredients of the system really are, and which physical processes merit detailed investigation by theorists. Among these would be photoionization equilibrium, particle acceleration, electron-positron pairs, relativistic MHD, and black holes.

The above diagram describes, I believe, how our subject actually progresses. We start off with only a very vague picture of what might be going on (or maybe several quite different alternatives); different kinds of odservations, and modelling of various individual ingredients, play complementary roles in revealing inconsistencies in our original viewpoint, and suggesting modifications, so that gradually a favoured scenario comes into consistently sharper focus.

\section{HOST GALAXIES, "FUZZ" AND QUASAR STATISTICS}

The relation of quasars to their host galaxies raises two questions. What is the relationship of quasars to radio galaxies (normally ellipticals) and to Seyferts (normally spirals)? Also, does a close companion or group membership enhance quasar activity? The latter has often been claimed, but the mechanism whereby a companion can trigger enhanced activity in the nucleus (rather than merely enhancing the star formation in the body of the galaxy) is still quite unclear.

A more general issue deserving further attention is the environmental impact of quasars. X-rays and UV from the quasar can heat, and even expel, gas in a surrounding galactic disc. Shock waves 
related to the extended radio structure could trigger star formation. For these reasons, and no doubt for others also, the observed fuzz is unlikely to resemble any normal undisturbed galaxies. Processes whereby the quasar reacts back on its surroundings may determine the life-cycle and the fuelling rate. Variability has of course been recorded over intervals from hours to years, but quasars may be equally variable on all longer timescales between 10 years and their total active lifetime. Moreover such variability could lead to transformations between different types of active galaxies (quasars $\rightarrow$ Seyferts, or quasars $\longleftrightarrow$ BL Lacs).

Let us turn now from individual objects to the properties of the collectivity of quasars. The energy output from quasars is known to within a factor of order 2: it is $\sim 3000 \mathrm{M}_{\odot} \mathrm{c}^{2}$ per $\mathrm{Mpc}^{3}$, about half coming from quasars with apparent magnitudes in the range $19-21$. Galaxies contribute $\sim 10^{5}$ in the same units, and the microwave background $\sim 7.5 \times 10^{6}$. So, even though quasars may influence their host galaxies, they are collectively rather modest contributors to the cosmic energy budget, because of their low space density. They may nevertheless have a crucial cumulative effect on the entire intergalactic medium, because their energy emerges largely in forms such as an ionizing continuum and high velocity jets and winds.

The prime era of quasar activity is at $z=2$ or 3 . It is from these redshifts that most of the quasar background light originates. The population thereafter decays on a timescale of order $t_{\text {Evo }}=2 x$ $10^{9}$ years. This is, however, merely an upper limit to the lifetime to of each object: many generations of individual quasars could be born, and could die, in the period over which the population declines.

Several important numbers depend on what $t_{0}$ actually is: the mass and the number of quasar remnants, the ratio of their luminosity to the Eddington limit, and the issue of whether the broadline region can be gravitationally bound.

The following table brings out these points. It contrasts two hypotheses: (i) that there was, in effect, only one generation of quasars, which were long-lived and massive; versus (ii) that there were $\sim 50$ generations of quasars, so that their individual masses (for a given efficiency, need not have built up to such high values, and quasar remnants would be more numerous.

$t_{Q} \cong t_{\mathrm{EVo}}$

$M=2.5 \times 10^{9} \varepsilon_{0.1} M_{\odot}$

$\mathrm{L} \ll \mathrm{L}_{\mathrm{Ed}}$

Broad-line regions gravitationally bound

Very massive remnants in 2\% of galaxies

$$
\begin{aligned}
& t_{Q} \cong 4 \times 10^{7} \text { yrs } \cong 0.02 t_{\text {Evo }} \\
& M=5 \times 10^{7} \varepsilon_{0.1} M_{\odot} \\
& L \cong L_{E d} \varepsilon_{0.1}
\end{aligned}
$$

Broad-line region not gravitationally bound

$\sim 10^{8} M_{\odot}$ remnants in most bright galaxies 
In this rough table, $\varepsilon_{0.1}$ is the efficiency measured in units of 0.1 . Clearly the z-dependence of the quasar population is related to galactic evolution, though we cannot yet quantify how. What other handle do we have on what the universe was like at redshift $z=3$, when it was perhaps only $1 / 8$ th its present age? Ordinary galaxies offer some clues. Cosmologists tell us that after the universe had expanded for about $10^{6}$ years, the fireball cooled down below $3000^{\circ}$, and the primaeval black body radiation shifted into the infrared. The universe then experienced a"dark age", which persisted until the first bound systems formed, igniting localised nuclear or gravitational power sources. When this "first light" occurred depends on the specific cosmogonic scenario. It could be at redshifts much greater than 2 , but need not be.

There are now, indeed, firmer reasons for believing that galaxy formation was still going on at $z=2$ or 3 . This reappraisal comes from evidence of dark halos around galaxies extending out to $r$ $\cong 100 \mathrm{kpc}\left(r_{100} \cong 1\right)$. If galaxies extended only out to $10 \mathrm{kpc}$, they could have formed on a timescale of $10^{8}$ years, at $z>10$. However, it is different if their massive halos extend out to $\gtrsim 100 \mathrm{kpc}$. The discs of galaxies could have formed from tidally-torqued material falling in,

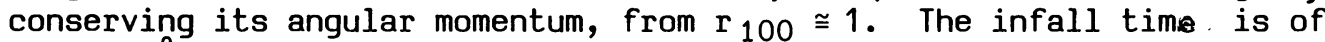
order $10^{9} r_{100}$ years, implying that infall from the halo, and disc formation, cannot be completed until the age of the universe is $2 \times$ $10^{9}$ years (i.e. $z=2$ or 3 ) for $r_{100} \equiv 1$.

When the universe was $\$ 20 \%$ its present age, the host galaxies, whose discs were still in the process of forming, would have been very different. We would expect more uncondensed or infalling gas to be present, implying that the fuzz should be more conspicuous, because it would more effectively scatter or reprocess the quasar light.

Is a high-z cutoff related to the formation of galaxies with welldefined nuclei? Or do higher $z$ quasars exist, shrouded by gas or dust in the host galaxy and therefore unrecognised?

\section{QUASARS AS PROBES}

An aspect of quasar studies that has burgeoned over the last few years, partly because it is in no way stymied by our confusion about their intrinsic properties, is their use as probes of the intervening medium: absorption by gas along the line of sight, and gravitational lensing by compact or massive bodies. Quasars allow us to probe 80 or 90\% of cosmic history - a period spanning the later stages of galaxy formation, and the contraction of clusters.

\section{Absorption lines: intervening gas clouds}

The statistics of the inferred absorbing clouds yield information on the $z$-dependence of the co-moving density of the clouds, the distribution of column densities, and velocity correlations. On the basis of such evidence one can begin to address the physics of individual clouds. In particular, how are they confined? Is it by the pressure of an external hot diffuse medium, or are they gravitationally bound? 
What are the sizes and shapes of the clouds? How do they relate to galaxies or protogalaxies?

Gravitational lensing by galaxies and "dark matter"

Lensing by the overall mass concentrations in galaxies, halos, and clusters yields multiple images on scales $\sim 1$ arcsec. Several such cases have been found over the last few years. Additionally, minilensing by individual compact objects of mass $M_{l}$ (stars, "Jupiters", or black holes in halos yields images separated by scales $\sim 10^{-6}\left(\mathrm{M}_{l} / M_{\odot}\right)^{\frac{1 / 2}{2}}$ arcsec. Even if these images cannot be optically resolved, they can contribute to variability and yield magnification.

Although lensing offers an important probe of the intervening medium, it is merely a confusing complication if we are trying to infer the intrinsic properties of quasars, such as the luminosity function. It is as though we view the remote universe through frosted glass. If the intrinsic luminosity function were very steep, magnified objects could preponderate in magnitude-limited surveys of high $z$ quasars.

\section{FINAL COMMENTS}

These sketchy and impressionistic comments - less a smorgasbord, I fear, than a dog's breakfast - paid no heed to the historical development of quasar studies. But the way things actually happened was really far from optimal - we were given many clues, but in a confusing order. It's ungracious to the discoverers of quasars to lament, with hindsight, the insight and resourcefulness they displayed in 1963. But if quasars had only been discovered in (say) 1973 - when we already knew more about lower-level activity in galactic nuclei (Seyferts, etc), and when pulsars and $X$-ray binaries had convinced us that gravitational energy could be channelleld efficiently into radiation - quasars would never have seemed 'sui generis', and theoretical ideas would have evolved less waywardly to their present state.

We shall be reminded later this week that there is not yet complete unanimity on even the most basic aspects of quasars. Though most astrophysicists are impressed that the growing body of data, when "conventionally" interpreted, meshes consistently into an overall cosmological and cosmogonic scheme, a few are convinced that some apparent anomalies demand a new (non-cosmological) mechanism for redshifts. The "dissidents", professing surprise that their claims meet persistent scepticism, sometimes attribute this to a conservative intellectual bias in the astronomical community - a blinkered reluctance to entertain fundamentally new ideas. But we sceptics - some of us, anyway - have, if anything, the opposite bias. The prospect that astronomers might discover some fundamentally new physics is a seductive one, and nothing could be more gratifying - and do more to render this a memorable conference - than the presentation of some novel and really clinching evidence for anomalies. Most of us might lay high odds against this happening; but let's hope nevertheless that 
the overall progress to be reported this week, along a broad front, will oblige Dr Woltjer drastically to update the draft of his concluding remarks which he doubtless already has on file.

\section{REFERENCES}

Brinkman, W. and Trumper, J. (eds) 1984, 'X-ray and UV emission from Quasars and Active Galactic Nuclei' (Publ. Max Planck Institut, München).

Dyson, J.E. (ed) 1985, 'Active Galactic Nuclei' (Manchester University Press).

Giuricin, G., Mardirossian, F. and Mezzetti, M. (eds) 1986, 'Structure and Evolution of Active Galactic Nuclei' (Reidel, Dordrecht).

Miller, J. (ed) 1985. 'Astrophysics of Active Galaxies and QuasiStellar Objects' (University Science Books, California).

Robinson, I., Schild, A. and Schucking, E.L. (eds) 1964, 'QuasiStellar Sources and Gravitational Collapse' (University of Chicago Press).

Swings, J.P. (ed) 1983, 'Quasars and Gravitational Lenses': Proc. 24th Liege Internat. Colloquium (Universite de Liège, Belgium).

\section{DISCUSSION}

Cowsik : Could you comment on Quasars as probes of dark matter ?

Rees : If the dark matter consists of individual compact objects - low mass faint stars or black holes - then "minilensing" is expected, as we shall I'm sure, learn from later speakers. The multiple images of QSOs which are interpreted as lensing by galactic-mass objects are of course a probe for massive halos in the lensing galaxies. Moreover, it seems that the $M / L$ of some lensing objects is exceptionally high, and this raises the interesting question of whether there may be a population of "failed galaxies" - massive halos without luminous cores. Such objects are expected in some models of galaxy formation, and gravitational lensing would be an important probe.

Kembhavi : Couldn't a large number of quasars at high redshift shrouded by young galaxies produce bumps in the background radiation at various wavelengths?

Rees : Yes. In particular, hard X-rays would not be absorbed by gas in a young galaxy. Even if we cannot directly detect objects of the highest $z$, background limits can certainly offer constraints on their collective properties.

Kundt : Could you comment on possible connections between QSO activity and the activity of young stellar objects (both classes of sources

appear to produce superacalc jets.) ? 
Rees : The velocities are of course much lower in the protostellar objects, may be because the gravitational potential wells are shallow. Your question also relates to the question of the collimation mechanism, which indeed may be similar in these two contexts. It's important to remember that we have no direct evidence that collimation in AGNs occurs on scales below about one parsec.

Burbidge : You have set the stage by talking about the "host galaxy". I would like to point out that there is no unambiguous evidence for galaxies around QSOs, and if they were there, the effect of the QSO might well make them abnormal. Thus in principle it may be difficult to prove that QSOs are embedded in host galaxies.

Rees : I agree.

Thakur : Validity of GTR has been tested and verified only in the weak field approximation but not for the strong fields. How can the models of the prime mover based on black holes be relied upon, especially when the singularity invovled does not appear to be physical and when the laws of physics do not hold good at the singularity?

Rees : A motivation for developing detailed models is the hope that this may reveal a diagnostic of the precise metric, and thereby permit some test of GTR, which has indeed, as you imply, only so far been validated when fields are weak. (However, I don't think our ignorance about physics near the singularity is an impediment. This singularity lies within the event horizon, and is irrelevant to observational phenomena. Analogously, one has confidence in the theory of atomic structure, despite our ignorance of subnuclear structure within individual protons.)

Thakur : Accretion discs around black holes have been suggested as the sources of energy from quasars and AGNs. However, these models appear to give steady out-flow of energy whereas violent activities have been observed in the quasars and AGNs. How can those contrary facts be reconciled?

Rees : Theorists have concentrated on stationary flow patterns in accretion flows. However, one would realistically expect the phenomena to be irregular on a whole range of timescales.

Segal : This was a very coherent and comprehensive account of quasar phenomena, but in part, and especially as regards evolution and differences between quasars at various redshifts, it appears as model-dependent. Is there any direct observational evidence for evolution, or is this simply a corollary to interpretation in a Friedman cosmology ?

Rees : It depends on assuming a Friedman-type model, as you rightly imply. 